\title{
A closed-circuit anesthesia ventilator facilitates significant reduction in sevoflurane consumption in clinical practice
}

\author{
Timur Özelsel, MD $\cdot$ Seok Hee Kim, RPh $\cdot$ Saifee Rashiq, BM BS · \\ Ban C. H. Tsui, MD
}

Received: 27 March 2015/Revised: 21 August 2015/Accepted: 1 September 2015/Published online: 11 September 2015

(c) Canadian Anesthesiologists' Society 2015

\section{To the Editor,}

In theory, closed-circuit anesthesia is the most efficient and economical way to deliver general anesthesia with a volatile anesthetic. Nevertheless, studies evaluating the Dräger Zeus ${ }^{\circledR}$ Infinity ${ }^{\circledR}$ Empowered (IE) ventilator - the only closed-circuit ventilator available in North America have generated mixed results. ${ }^{1-5}$ Most studies evaluating the closed-circuit machine and semi-closed-circuit machines have compared the automated mode of the Zeus IE with the traditional semi-closed-circuit run by the anesthesiologist under optimal conditions. Our goal was to investigate whether the closed-circuit ventilator offers a reduction in the consumption of volatile agent without requiring anesthesiologists to change their practice habits.

Seven staff anesthesiologists were recruited to use the Zeus IE and two semi-closed-circuit ventilators (Dräger Primus ${ }^{\circledR} 4$ and Dräger Fabius ${ }^{\circledR}$ GS) for one week each while running their regular surgical lists. Under the study protocol, each anesthesiologist administered general anesthesia according to their usual practice for three weeks. Specifically, they followed their usual practices with respect to vaporizer settings and fresh gas flow rates when using the semi-closed-circuit machines and ran the case in auto control mode with a closed-circuit when using the Zeus IE ventilator. There was no preferential selection of surgical cases during the three-week study period. Every anesthesia machine was used with its most current software version. The only requirement for each case was to use

T. Özelsel, MD $(\varangle) \cdot$ S. H. Kim, RPh · S. Rashiq, BM BS ·

B. C. H. Tsui, MD

Department of Anesthesiology and Pain Medicine, University of

Alberta, Edmonton, AB, Canada

e-mail: ozelsel@ualberta.ca sevoflurane in oxygen-enriched air as the sole volatile anesthetic.

Consumption of volatile anesthetic was recorded for each anesthesiologist and anesthesia machine. Data were compared using general linear modelling and Duncan's post hoc test. The consumption data for the Zeus IE and Primus ventilators were recorded by the machine itself. For the Fabius, which does not display the amount of volatile anesthetic consumed, we weighed the amount needed to refill the vaporizer and then divided the weight by the density of sevoflurane $\left(1.52 \mathrm{~g} \cdot \mathrm{cm}^{-3}\right)$ to obtain the volume.

The seven anesthesiologists in the study delivered anesthesia for 240 cases in total during the study period. Case-specific hourly sevoflurane consumption rates were obtained for all 240 cases. All anesthesiologists used fresh gas flows in the range of $0.5-1.0 \mathrm{~L} \cdot \mathrm{min}^{-1}$. The Zeus IE machine used significantly less volatile anesthetic [mean (SD), $8.1(1.5) \mathrm{mL} \cdot \mathrm{hr}^{-1}$ ] than the two semi-closed-circuit machines [Fabius, $12.0(2.1) \mathrm{mL} \cdot \mathrm{hr}^{-1}$; Primus, 12.1 (3.4) $\left.\mathrm{mL} \cdot \mathrm{hr}^{-1} ; \quad P=0.01\right]$ (Figure). Consumption between practitioners did not differ $(P=0.11)$, and there was no statistically significant interaction between practitioner and machine.

We examined our own institution as an example when considering the financial implications. The University of Alberta Hospital has over 650 beds, and our anesthesia department was estimated to provide $26,726 \mathrm{hr}$ of general anesthesia in 2014. Sevoflurane costs $\$ 180.00$ CAD per $250 \mathrm{~mL}$ bottle at our hospital $(\$ 0.72$ per $\mathrm{mL})$. Assuming we used sevoflurane exclusively with a closed-circuit machine for all surgeries requiring volatile anesthetic, the estimated annual savings would be significant $(\$ 84,859.92$ CAD vs an all-Fabius GS fleet under the same parameters and $\$ 69,850.80$ CAD vs an all-Primus fleet). Nevertheless, considering the significant expense of the Zeus IE machine 


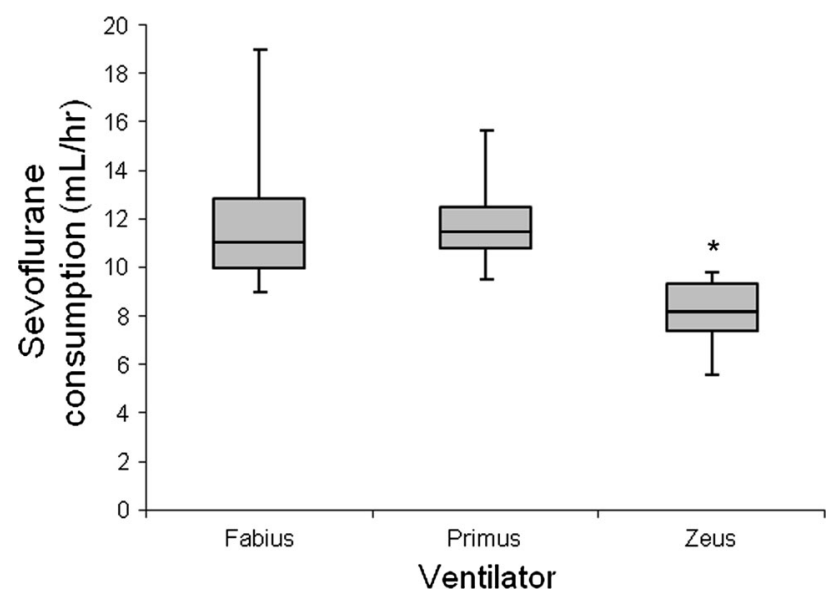

Figure The Zeus IE anesthesia machine consumes significantly less sevoflurane compared with the semi-closed circuit Fabius GS and Primus 4 machines. Grey box indicates first to third quartile values, bars indicate range, and line within the grey box indicates median value. $* P=0.01$ compared with both Fabius and Primus

( $\sim \$ 100,000$ CAD), cost savings could potentially be achieved simply by educating staff and reducing fresh gas flow to a bare minimum on the semi-closed circuit anesthesia machines.

In summary, although our study was limited by a small sample size, we demonstrated in a real-life clinical situation that a closed-circuit ventilator can significantly reduce consumption of a volatile anesthetic agent in comparison with semi-closed-circuit ventilators. This was previously accepted as only a theoretical advantage of closed-circuit systems. This reduction in consumption has implications for reducing not only healthcare costs but also environmental waste.

Conflicts of interest None declared.

\section{References}

1. De Cooman S, De Mey N, Dewulf BB, et al. Desflurane consumption during automated closed-circuit delivery is higher than when a conventional anesthesia machine is used with a simple vaporizer- $\mathrm{O}_{2}-\mathrm{N}_{2} \mathrm{O}$ fresh gas flow sequence. BMC Anesthesiol 2008; 8: 4 .

2. Park $J Y$, Shin HW, Jeon SK, et al. A comparison of consumption and recovery profiles according to anaesthetic circuit mode using a new multifunctional closed-circuit anaesthesia system during desflurane anaesthesia: a clinical study. J Int Med Res 2010; 38: 160-8.

3. de Monte A, Vecil M, di Stefano C, Zorzi F, Saltarini M. Low flow, minimal flow and closed circuit system inhalational anesthesia in modern clinical practice. Signa Vitae 2008; 3(Suppl 1): S33-6.

4. De Cooman S, Hendrickx JF, Peyton PJ, Demeere JL, De Wolf $A M$. Agent consumption with the Zeus(R) in the automated closed circuit anesthesia mode with $\mathrm{O}_{2} /$ air mixtures. BMC Res Notes 2014; 7: 469.

5. Lokoff A, Lokoff P. Volatile gas consumption after introduction of the Drager Zeus ${ }^{\circledR}$ anesthesia machine. Can J Anesth 2014; 61: 680-1. 